\title{
Nogo-A inhibits the migration and invasion of human malignant glioma U87MG cells
}

\author{
SHU-GUANG JIN ${ }^{1 *}$, HYANG-HWA RYU ${ }^{2 *}$, SONG-YUAN LI², CHUN-HAO LI ${ }^{2}$, \\ SA-HOE LIM ${ }^{2,3}$, WOO-YOUL JANG ${ }^{3}$ and SHIN JUNG ${ }^{2,3}$ \\ ${ }^{1}$ Department of Neurosurgery, Ningbo No. 9 Hospital, Zhejiang 315010, P.R. China; \\ ${ }^{2}$ Brain Tumor Research Laboratory, ${ }^{3}$ Department of Neurosurgery and Brain Tumor Clinic and Gamma Knife Center, \\ and Chonnam National University Research Institute of Medical Sciences, Chonnam National University \\ Medical School and Hwasun Hospital, Hwasun 519-763, Republic of Korea
}

Received December 15, 2015; Accepted February 5, 2016

DOI: $10.3892 /$ or.2016.4737

\begin{abstract}
Nogo or reticulon-4 (RTN4), also known as neurite outgrowth inhibitor, is a member of the reticulon family of genes. Nogo-A, one of the three isoforms, is enriched in the central nervous system (CNS). The extracellular domain of Nogo-A, Nogo-66, has neurite growth inhibitory activity that is specific for neurons and is mediated by the Nogo receptor. However, most of its functions are not known yet. We investigated whether Nogo-A modulates the migration and invasion of a glioblastoma cell line, as well as the factors that have an effect on Nogo-A. The expression of Nogo-A was evaluated using western blotting and immunohistochemistry in human brain tumor specimens. U87MG cells were transfected with a sense-Nogo-A cDNA construct (U87-Nogo-A cells expressing Nogo-A) and an empty vector (U87MG-E cells not expressing Nogo-A). The migration and invasion abilities of these cells were investigated using simple scratch and Matrigel invasion assays. Morphologic and cytoskeletal changes were documented by confocal microscopy. The proliferation rate was estimated using doubling time assay. The effects of Nogo-A on Rho activity and phosphorylated cofilin were determined by a Rho activity assay and western blotting. Among primary brain tumors, Nogo-A expression was found in a higher percentage of oligodendrogliomas $(90.0 \%)$ compared with the percentage in the glioblastomas $(68.4 \%)$. In addition, the percentage in mixed gliomas was $42.9 \%$, while it was not expressed in pituitary adenomas or schwannomas. The migration and invasion
\end{abstract}

Correspondence to: Professor Shin Jung, Department of Neurosurgery, Chonnam National University Hwasun Hospital and Medical School, 322 Seoyang-ro, Hwasun-eup, Hwasun 58128, Republic of Korea

E-mail: sjung@jnu.ac.kr

${ }^{*}$ Contributed equally

Key words: malignant glioma, invasion, migration, U87MG cell line, Nogo-A abilities of the U87-Nogo-A cells were decreased compared with the control. In the U87-Nogo-A cell line, Rho activity and phosphorylated cofilin expression were also decreased and morphology became more flat in comparison with the U87MG-E cell line. Nogo-A may inhibit the migration and invasion of human malignant glioma cells via the downregulation of RhoA-cofilin signaling.

\section{Introduction}

Glioblastoma multiforme (GBM), the most aggressive glial tumor, is associated with a median survival rate of 9-12 months. Advances in the basic knowledge of cancer biology, as well as surgical techniques, chemotherapy and radiotherapy, have led to only a marginal improvement in the survival rate for $\operatorname{GBM}(1,2)$. Other well-tolerated and effective therapeutic approaches are clearly needed. In the present study, we aimed to identify a factor associated with tumor cell migration and invasion, which may provide a more effective treatment strategy.

Nogo or reticulon-4 (RTN4), also known as neurite outgrowth inhibitor, is a member of the reticulon family of genes, and is encoded by a single gene on human chromosome 2 (2p16) with 14 exons spanning a $75-\mathrm{kb}$ stretch, and 10 known splice variants (3). Nogo occurs in 3 major forms, Nogo-A, Nogo-B and Nogo-C, which are generated from alternate splicing (4). Nogo-A is the longest isoform based on transcript and protein level analyses, and is enriched in the central nervous system (CNS). However, the other abundant splice isoform, Nogo-B, is expressed more ubiquitously (5-7). Nogo-C is highly expressed in skeletal muscle, but is weakly expressed in the liver and kidney (8). All 3 splice isoforms have an $\mathrm{N}$-terminal domain of varying length, and share an identical C-terminal domain comprising a 66-amino acid loop (Nogo-66) flanked by two hydrophobic segments, followed by a short C-terminal hydrophilic stretch.

Nogo-A has been described as a myelin-associated neuronal growth inhibitory molecule that interacts with the Nogo receptor ( $\mathrm{NgR})$, and signaling appears to be related to the activation of Rho A (9). Rho is a member of the Ras superfamily of small GTP-binding proteins, and the mammalian Rho GTPase family currently consists of 3 major subfamilies: Rho 
(RhoA, RhoB and RhoC), Rac (Rac1, Rac2 and Rac3) and Cdc42 (Cdc42Hs and G25K). These subfamilies play a central role in diverse biological processes, such as actin cytoskeleton organization, microtubule dynamics, gene transcription, oncogenic transformation, cell cycle progression, adhesion and epithelial wound repair (10).

In brain tumors, Nogo-A was found to be expressed in oligodendroglioma and GBM in an immunohistochemical study (11) and was negatively related to the malignancy of oligodendroglial tumors $(12,13)$. Furthermore, Nogo was found to inhibit neurite outgrowth molecules (14). These previous studies suggest the possibility that Nogo-A is a negative regulator of the migration and invasion of glioma cells (15); nevertheless, the role of Nogo-A in brain tumors remains unclear. Thus, in the present study, we investigated whether Nogo-A can regulate the migration and invasion of malignant glioma.

\section{Materials and methods}

Cell lines and tissues. Human glioblastoma cell line U87MG was obtained from the Korean Cell Line Bank (Seoul, Korea). All cell lines were maintained in Dulbecco's modified Eagle's medium (DMEM) (Gibco-BRL, Grand Island, NY, USA) and supplemented with $10 \%$ fetal bovine serum (FBS) at $37^{\circ} \mathrm{C}$ in a humidified $95 \%$ air $/ 5 \% \mathrm{CO}_{2}$ atmosphere.

Brain tumor tissues (38 GBMs, 30 oligodendrogliomas, 7 mixed gliomas, 3 pituitary adenomas and 3 schwannomas) were obtained from the Chonnam National University Hwasun Hospital National Biobank of Korea. The specimens were snap-frozen in liquid nitrogen and stored at $-70^{\circ} \mathrm{C}$ until use.

Immunohistochemistry of Nogo-A. Immunohistochemistry was performed using the avidin-biotin complex method with a microprobe immuno/DNA stainer (Fisher Scientific, Pittsburgh, PA, USA). Paraffin-embedded blocks of formalin-fixed surgical specimens (20 GBM and 29 oligodendrogliomas obtained from the Chonnam National University, Hwasun Hospital National Biobank of Korea) were cut into $3-\mu \mathrm{m}$ thick sections with a microtome and placed on microscope slides. The sections were deparaffinized in xylene and treated with $3 \% \mathrm{H}_{2} \mathrm{O}_{2}$ in methanol for 20 min to block endogenous peroxidase activity. After washing several times with immuno/DNA buffer (Invitrogen, Carlsbad, CA, USA), the sections were incubated with $2 \%$ normal serum to block any non-specific binding, followed by incubation with a rabbit anti-Nogo-A polyclonal antibody (1:100 dilution; Santa Cruz Biotechnology, Santa Cruz, CA, USA) at $4^{\circ} \mathrm{C}$ overnight. A streptavidin horseradish peroxidase (Dako Cytomation, Denmark) detection system was applied to the capillary channels, followed by a $20-$ min incubation at $37^{\circ} \mathrm{C}$. After rinsing, the tissue sections were ready for the chromogen reaction with 3-amino-9-ethyl carbazole. The sections were counterstained with hematoxylin and mounted on universal mounts (Shandon, Pittsburgh, PA, USA). Finally, the coverslips were fixed to the slides with mounting solution.

Preparation of the plasmid containing human Nogo-A $c D N A$. Full-length Nogo-A cDNA was purchased from Open Biosystems (Huntsville, AL, USA) (clone ID 40148984), and cloned into the pcDNA3.1 (+) expression vector (Invitrogen) containing the cytomegalovirus promoter and the neomycin-resistance gene. The resulting pcDNA3.1 (+)-Nogo-A sequence completely matched the NCBI accession (BC 150182) and was used to transfect the U87MG cells.

Transfection procedure. U87MG cells were maintained under exponential growth conditions in DMEM supplemented with $10 \%$ FBS, in the absence of antibiotics. The optimum cell density for transfection is normally between 50 and $80 \%$ confluency for adherent cells. U87MG cells were transfected with the empty pcDNA3.1 (+) vector and pcDNA3.1 (+)-Nogo-A using Lipofectamine ${ }^{\mathrm{TM}} 2000$ transfection reagent (Invitrogen). These transfectants were designated as 'U87MG-E' and 'U87-Nogo-A', respectively. Plasmid DNA $(6 \mu \mathrm{g})$ and $6 \mu \mathrm{l}$ Lipofectamine 2000/serum-free media were added to the cells growing in serum-free media, according to the manufacturer's protocol. After a 5 -h incubation at $37^{\circ} \mathrm{C}$ in $5 \% \mathrm{CO}_{2}$, the transfection mixture was replaced with DMEM supplemented with $10 \%$ FBS. Forty-eight hours later, the medium was replaced with DMEM containing 10\% FBS and $800 \mu \mathrm{g} / \mathrm{ml} \mathrm{G418}$ and cultured in a $\mathrm{CO}_{2}$ incubator. The G418-resistant clones were isolated, and the level of expression of the Nogo-A protein was determined by western blot analysis. The stable transfectants were maintained in DMEM supplemented with $10 \%$ FBS and $400 \mu \mathrm{g} / \mathrm{ml} \mathrm{G} 418$.

Western blotting. Cell lines and brain tissues were lysed in lysis buffer [50 mM Tris (pH 8.0), 5 mM ethylene diamine tetraacetic acid, $150 \mathrm{mM} \mathrm{NaCl}, 0.5 \%$ deoxycholic acid, $0.1 \%$ sodium dodecyl sulphate, $1 \%$ NP-40, $1 \mathrm{mM}$ phenylmethylsulphonyl fluoride and $1 \mathrm{mg} / \mathrm{ml}$ protease inhibitor cocktail]. The protein concentrations were determined using the bicinchoninic acid (BCA) method (16). Subsequently, $50 \mu \mathrm{g}$ whole cell lysate was separated by $8 \%$ sodium dodecyl sulphate-polyacrylamide gel electrophoresis (SDS-PAGE) and transferred onto a polyvinylidene fluoride membrane (PALL Corp., Mexico). The membrane was then incubated for $2 \mathrm{~h}$ at room temperature in a solution of TBS-T [10 mM Tris- $\mathrm{Cl}$ (pH 8.0), $150 \mathrm{mM} \mathrm{NaCl}$ and $0.05 \%$ Tween-20] supplemented with 5\% non-fat dry milk, and probed overnight at $4{ }^{\circ} \mathrm{C}$ with primary antibodies against Nogo-A, cofilin and phosphorylated cofilin (Santa Cruz Biotechnology). The bound antibodies were visualized with an anti-rabbit secondary antibody (Jackson ImmunoResearch Laboratories, West Grove, PA, USA) conjugated to horseradish peroxidase using enhanced chemiluminescence reagents (Amersham Biosciences, Pittsburg, PA, USA). $\beta$-actin was used as an internal control and rat brain tissue was used as a Nogo-A-positive control.

Migration assay. For the comparison of motility between the U87MG-E and U87-Nogo-A cells, a simple scratch technique was performed. Cells were grown to confluency on a $60-\mathrm{mm}$ dish, and the medium was replaced with medium containing $5 \mathrm{mM}$ hydroxyurea to stop cell proliferation. After $24 \mathrm{~h}$, the cultures were scraped using a single-edged razor blade. Cells were washed twice with phosphate-buffered saline (PBS), placed in medium containing $5 \mathrm{mM}$ hydroxyurea, and incubated for $48 \mathrm{~h}$. Following this, the cells were washed twice 
with PBS, fixed with absolute alcohol, and stained with $0.1 \%$ toluidine blue. Six microscopic fields were evaluated for each wound injury. The number of cells migrating across the wound edge and the maximum distance migrated were determined in each field, and averaged for each injury. These experiments were repeated 3 times.

Matrigel invasion assay. Matrigel (reconstituted basement membrane; $25 \mathrm{mg}$ ) was dried on a polycarbonated filter (polyvinylpyrrolidone-free; Nucleopore Whatmann, UK). The cells were harvested by brief exposure to $1 \mathrm{~mm} / 1$ EDTA, washed with DMEM containing $0.1 \%$ bovine serum albumin, and added to a Boyden chamber $\left(1 \times 10^{4}\right.$ cells). Cells were incubated for $24 \mathrm{~h}$ at $37^{\circ} \mathrm{C}$ in a humidified atmosphere of $95 \%$ air and $5 \% \mathrm{CO}_{2}$. The cells that transversed the Matrigel layer and attached to the filter were stained with Hemacolor (Darmstadt, Germany) and counted in five randomized fields. The results are expressed as the mean $\pm \mathrm{SE}$ of 3 independent experiments.

Rho activity assay. According to the manufacturer's protocol included in the Rho activity assay kit (Millipore, Billerica, MA, USA), U87MG-E and U87-Nogo-A cells were cultured to $\sim 85-90 \%$ confluency, the culture medium was removed, and the cells were rinsed twice with ice-cold Tris-buffered saline (TBS). Ice-cold $\mathrm{Mg}^{2+}$ lysis/wash buffer (MLB) $25 \mathrm{mM}$ HEPES (pH 7.5), $150 \mathrm{mM} \mathrm{NaCl}, 1 \%$ Igepal CA-630, $10 \mathrm{mM} \mathrm{MgCl}$, $1 \mathrm{mM}$ EDTA and $2 \%$ glycerol containing $1 \mathrm{mM}$ phenylmethylsulfonyl fluoride (PMSF)] was added to the rinsed cells in plates on ice. The detached cells were collected and lysed in microfuge tubes on ice, the tubes were incubated for $20 \mathrm{~min}$ at $4^{\circ} \mathrm{C}$ with agitation, and then centrifuged at $14,000 \mathrm{x}$ g for $5 \mathrm{~min}$ at $4^{\circ} \mathrm{C}$. The supernatant was removed. The minimum protein concentration was $\sim 8 \mathrm{mg} / \mathrm{ml}$. A $500 \mathrm{ml}$ aliquot of each cell extract was placed in a microfuge tube, $40 \mathrm{ml}$ of the Rho assay reagent slurry was added, and the reaction mixture was incubated for $45 \mathrm{~min}$ at $4^{\circ} \mathrm{C}$ with gentle agitation. The agarose beads were pelleted by brief centrifugation (10 sec at $14,000 \mathrm{x} \mathrm{g}$ at $4^{\circ} \mathrm{C}$ ), and the supernatant was removed and discarded. The beads were washed 3 times with $0.5 \mathrm{ml} 1 \mathrm{X}$ MLB. The beads were mixed gently, pelleted and the supernatant was removed. The agarose beads were resuspended in $25 \mathrm{ml} 2 \mathrm{X}$ Laemmli reducing sample buffer and boiled for $5 \mathrm{~min}$. The beads were then pelleted by centrifugation. The supernatant and the agarose pellets were mixed and then loaded for Rho western blot analysis. In parallel, a sample that was not treated with the Rho assay reagent slurry was loaded to determine the total Rho amount.

Doubling time of stable transfectants. The cells were seeded at $3 \times 10^{4}$ cells on 6 -well culture dishes. After serum starvation for $48 \mathrm{~h}$, the cells were counted every $24 \mathrm{~h}$. The cells were trypsinized and the number of viable cells was counted with a hemocytometer. The doubling-time was calculated from the cell growth curve over 4 days using the following equation: Doubling time $=($ final time - initial time $) \times[\log 2 / \log ($ final cell number) - $\log$ (initial cell number)].

Immunofluorescence confocal microscopy. The cells were cultured on coverslips in 35-mm dishes until subconfluency was reached, washed with PBS, and fixed with $4 \%$ parafor- maldehyde for $10 \mathrm{~min}$. After washing (3-5 times) in PBS, the cells were treated with $0.1 \%$ Triton X-100 for $5 \mathrm{~min}$ at room temperature, and washed again 3-5 times with PBS. The cells were incubated with anti-vimentin (BD Pharmingen, San Diego, CA, USA) in a humid chamber for $1 \mathrm{~h}$, and then with Alexa 488-conjugated goat anti-mouse antibody (Molecular Probes, Eugene, CA, USA) for $40 \mathrm{~min}$. For actin staining, Rhodamine-conjugated phalloidin (Molecular Probes) was used. The coverslips were mounted on slides with Immuno Mounts (Shandon). Confocal microscopy was performed using an FV10-ASW, version 1.7 confocal laser scanning biological microscope (Olympus, Tokyo, Japan) equipped with an UPlanSApo 60x/1.35 oil objective lens. The confocal images were acquired using FluoView FV1000 software.

Data analysis. The comparison of the nucleotide sequence of the cDNA (purchased) with the registered sequence in GeneBank was carried out using the BLAST algorithm. The statistical significance of the cell distance and cell number was measured using the Mann-Whitney U test, and the doubling time by repeated measures ANOVA. $\mathrm{P}<0.05$ was considered to indicate a statistically significant result. Statistical analysis was performed using SPSS (version 12.0 for Windows; SPSS, Inc., Chicago, IL, USA).

\section{Results}

Expression of Nogo-A in glial tumors and Nogo-A transfectants. The expression of Nogo-A was examined using immunohistochemistry (IHC) and western blotting. The expression of Nogo-A was exhibited in 90, 68.4 and $42.9 \%$ of oligodendrogliomas, glioblastomas and mixed gliomas from patients, respectively. However, schwannomas and pituitary adenomas did not express Nogo-A (Fig. 1). Similarly, Nogo-A was expressed in a higher percentage of oligodendrogliomas $(79.3 \%)$, compared with the glioblastomas $(35 \%)$ by IHC (Fig. 2). The brown-positive signals were mainly distributed in the cytoplasm. These results are summarized in Table I.

Endogenous Nogo-A content. To assess the effect of Nogo-A on proliferation, migration and invasion, U87MG malignant glioma cells, not expressing Nogo-A, were transfected with an empty vector ('U87MG-E') and a sense Nogo-A cDNA construct ('U87-Nogo-A'). The best clone among the transfectants was selected using western blotting. Nogo-A was highly expressed in the U87-Nogo-A cells, compared with its level in the U87MG parental and U87MG-E cells (Fig. 3).

Proliferation rate. We examined the effect of Nogo-A on cell proliferation. The doubling times in the U87MG-E and U87-Nogo-A cells were 37.1 and $29.2 \mathrm{~h}$, respectively, showing statistical significance $(\mathrm{P}<0.001 ;$ Fig. 4 and Table II).

Effect of Nogo-A on migration ability; a simple scratch test. The motility of the cell lines was detected using a simple scratch technique. The difference in proliferation between the two cell lines may affect the results of the in vitro motility assay. To exclude this factor, we pretreated the cells with hydroxyurea, which causes cell cycle arrest. As shown in 

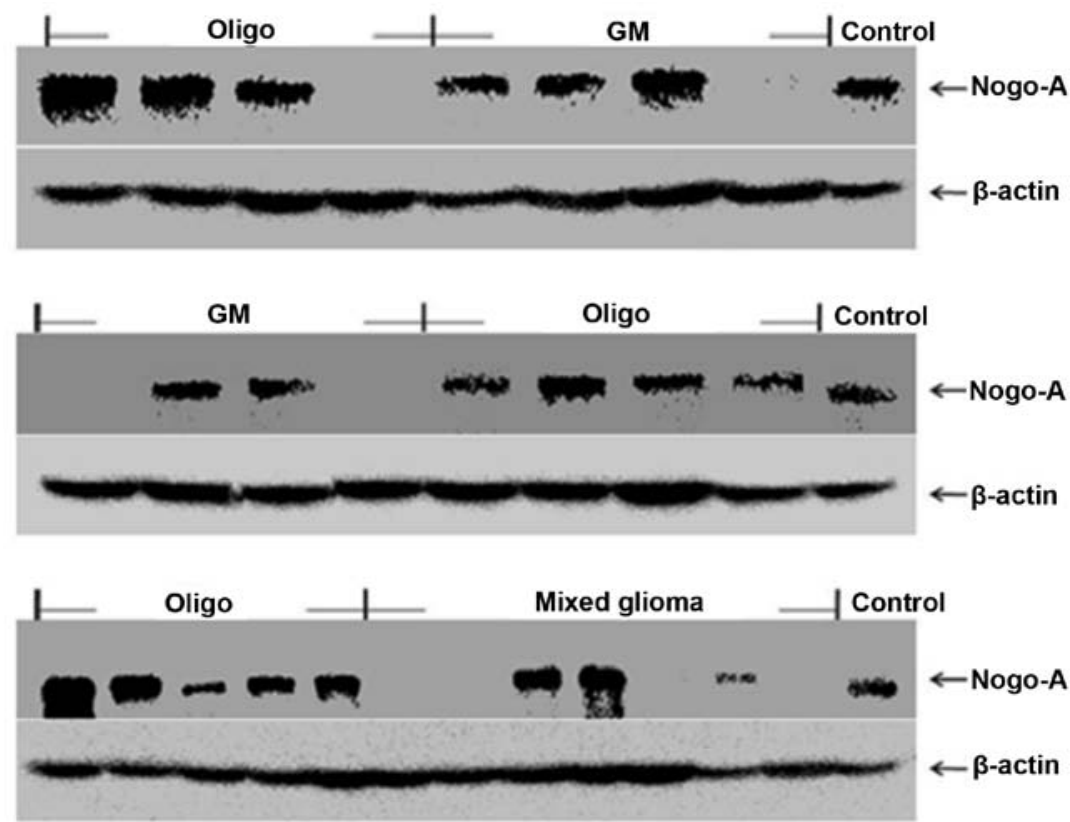

Figure 1. Expression of Nogo-A in human glial tumors using western blotting. Nogo-A was expressed in the oligodendrogliomas (Oligo), glioblastomas (GM), and mixed gliomas. $\beta$-actin was used as an internal control.
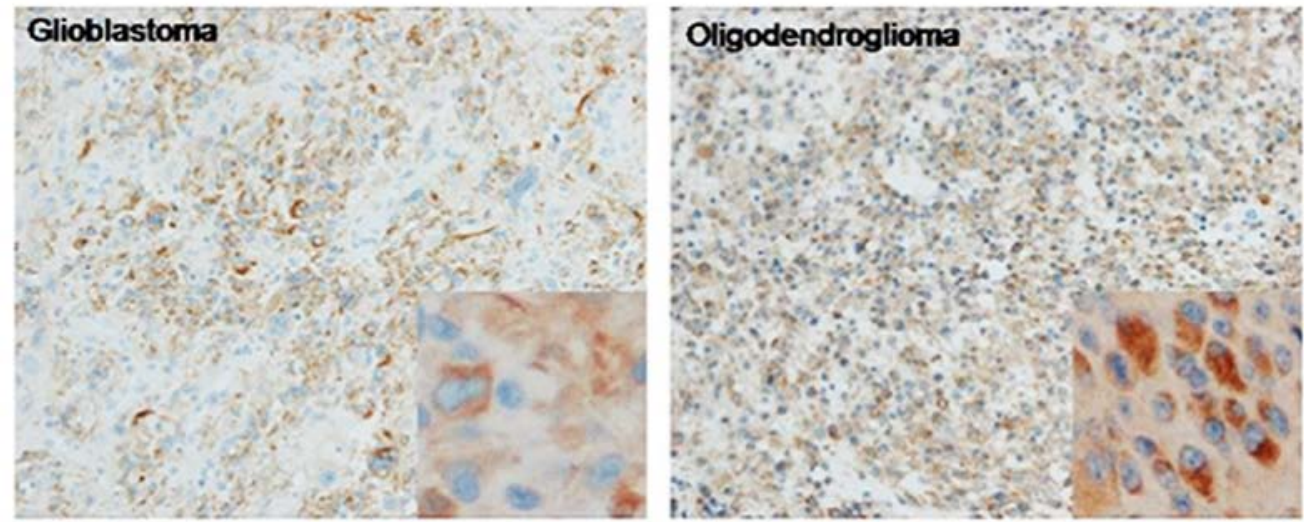

Figure 2. Expression of Nogo-A in human glial tumors using immunohistochemistry. Nogo-A was expressed in the cytoplasm of the tumor cells. Oligodendrogliomas showed a higher expression of Nogo-A and a stronger intensity compared with the glioblastomas. The insets show a higher magnification (x200).

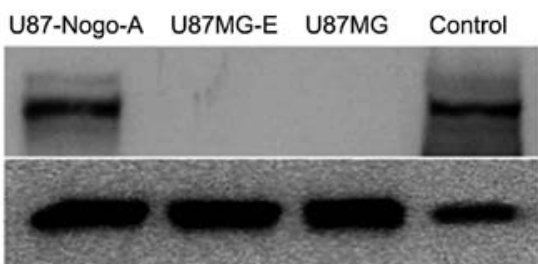

Figure 3. Selection of the best clone among the U87MG cells transfected with sense Nogo-A construct by means of western blotting. U87-Nogo-A cells showed a strong band of Nogo-A while U87MG and U87MG-E cells did not show bands.

Fig. 5 and Table III, the total number of cells that migrated from the wound in the U87MG-E and U87-Nogo-A cell lines was $311 \pm 18.4$ and $163.3 \pm 31$, respectively. In addition, the maximum distance of migration from the wound in the
Table I. Nogo-A expression in glial tumors.

\begin{tabular}{|c|c|c|}
\hline Histology & $\begin{array}{l}\text { Western blotting } \\
\mathrm{n} / \text { total }(\%)\end{array}$ & $\begin{array}{c}\text { Immunohistochemistry } \\
\text { n/total (\%) }\end{array}$ \\
\hline Glioblastoma & 26/38 (68.4) & $7 / 20(35.0)$ \\
\hline Oligodendroglioma & 27/30 (90.0) & 23/29 (79.3) \\
\hline Mixed glioma & 3/7 (42.9) & \\
\hline Pituitary adenoma & $0 / 3(0)$ & \\
\hline Schwannoma & $0 / 3(0)$ & \\
\hline
\end{tabular}

U87MG-E and U87-Nogo-A cells was 1,100 and $700 \mu \mathrm{m}$, respectively. These results suggest that the expression of Nogo-A in the U87MG cells is inversely associated with the migration ability $(\mathrm{P}=0.005)$ of the tumor cells. 


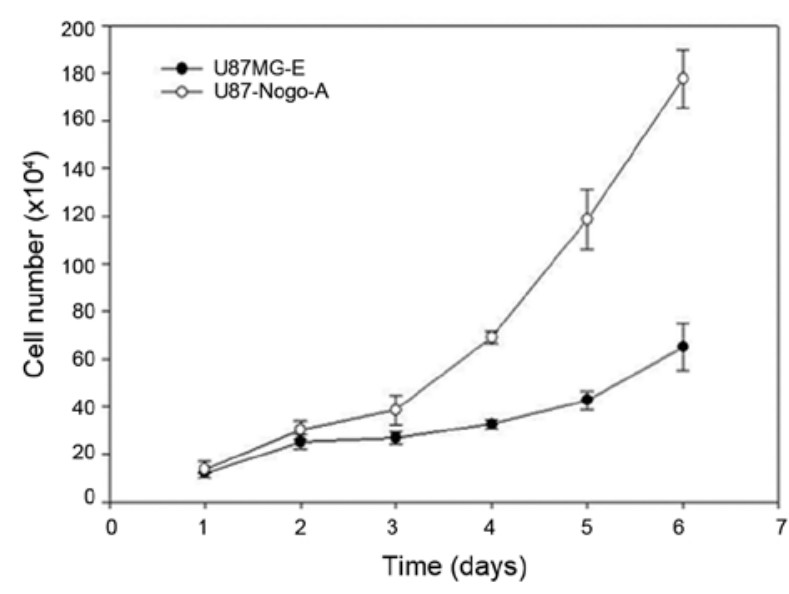

Figure 4. Determination of the growth rates using doubling time assay. U87MG-E and U87-Nogo-A cells displayed a doubling time of 37.1 and $29.2 \mathrm{~h}$, respectively $(\mathrm{P}<0.001)$.

Table II. Comparison of the growth rate between the control and Nogo-A-overexpressing cells.

\begin{tabular}{lc}
\hline Cell line & Doubling time $(\mathrm{h})$ \\
\hline U87MG-E & 37.1 \\
U87-Nogo-A & 29.2 \\
\hline
\end{tabular}
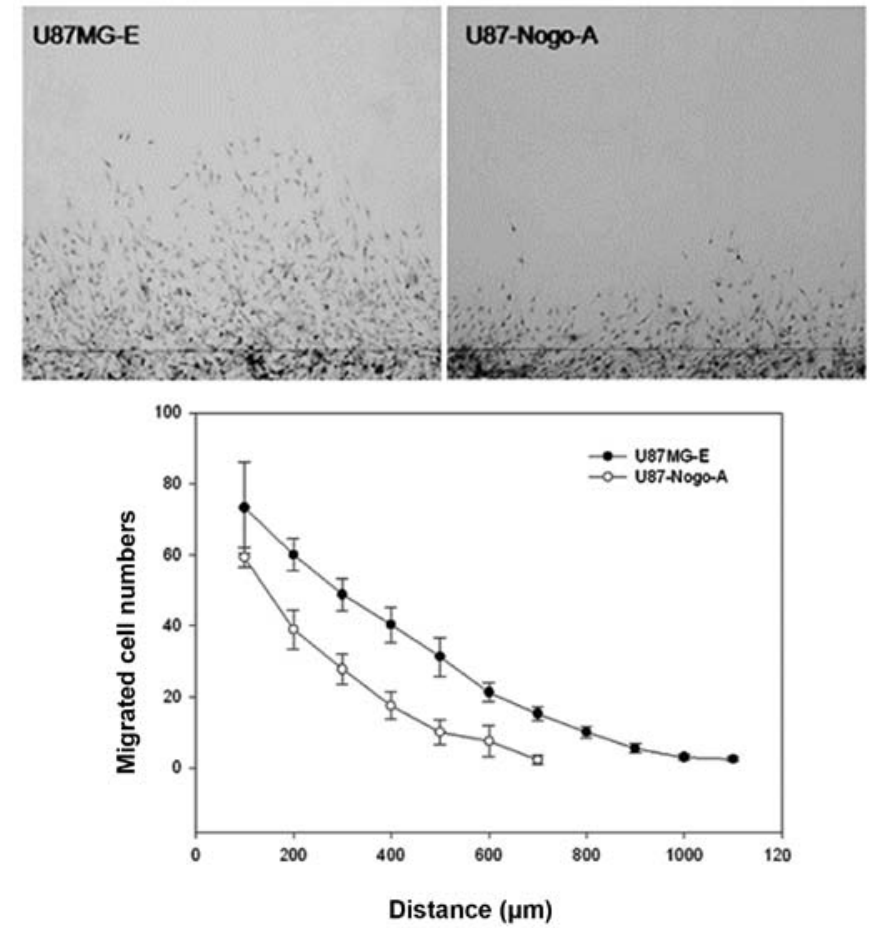

Figure 5. Effect of Nogo-A on the migration of U87MG cells by means of a scratch assay. The result evaluated total cell number and maximum distance of migration from the wound edge. The difference between U87-Nogo-A and U87MG-E (control) cells showed statistical significance $(\mathrm{P}=0.005)$.

Effect of Nogo-A on invasion; the Boyden chamber assay. The effect of Nogo-A on the invasion of tumor cells was examined
Table III. The results of the scratch test.

\begin{tabular}{lcc}
\hline Cell line & Cell number & Maximum distance $(\mu \mathrm{m})$ \\
\hline U87MG-E & $311 \pm 18.4$ & 1,100 \\
U87-Nogo-A & $163.3 \pm 31$ & 700 \\
\hline
\end{tabular}

The test evaluated total migrated cell number and the maximum distance migrated from the wound edge.
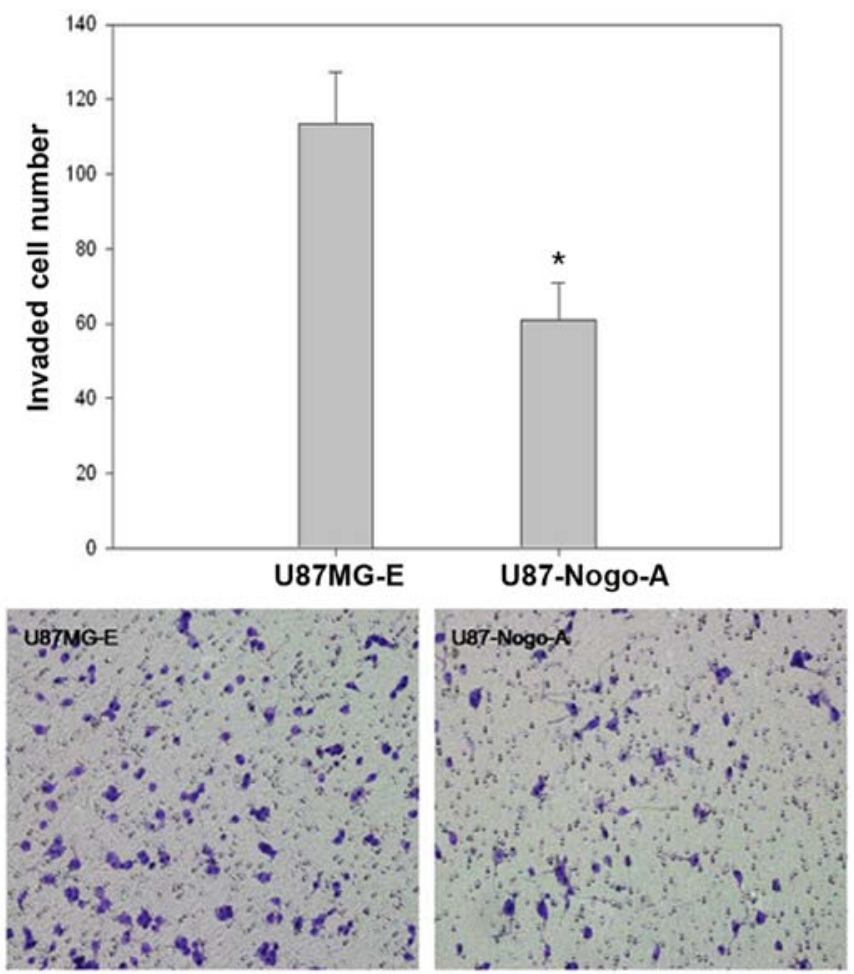

Figure 6. Effect of Nogo-A on the invasion of U87MG cells by means of a Matrigel assay. The total number of cells that invaded the Matrigelcoated membrane were counted. The difference between U87-Nogo-A and U87MG-E (control) cells showed statistical significance $(\mathrm{P}<0.001)$.

Table IV. Results of the Matrigel assay.

\begin{tabular}{lc}
\hline Cell line & Cell number \\
\hline U87MG-E & $113.5 \pm 13.8$ \\
U87-Nogo-A & $60.9 \pm 9.9$
\end{tabular}

Total number of cells that invading the Matrigel-coated membrane were counted.

using a Boyden chamber assay. This method is for quantitative analysis of the invasion ability of tumor cells. It is estimated by counting the number of cells invading through the Matrigelcoated membrane. The total number of U87MG-E and U87-Nogo-A cells was $113.5 \pm 13.8$ and $60.9 \pm 9.9$, respectively. Invasiveness of U87MG cells was significantly decreased by overexpression of Nogo-A ( $\mathrm{P}<0.001$; Fig. 6 and Table IV). 


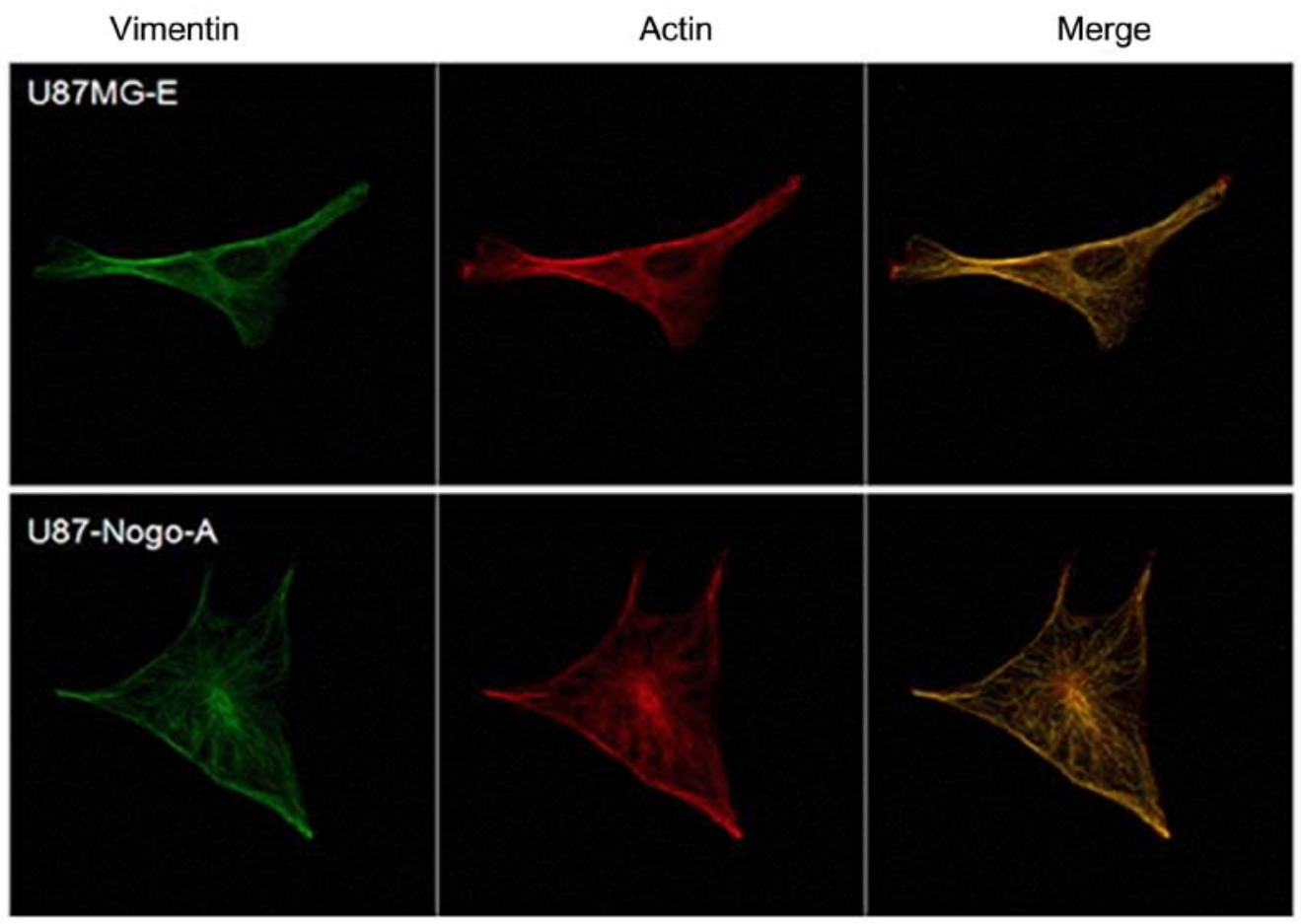

Figure 7. Immunofluorescence staining of cytoskeletal proteins. Cytoskeletal change in the U87-Nogo-A cells compared with that observed in the U87MG-E cells. The patterns of the following elements of the cytoskeleton are presented: actin (red) and vimentin (green).

A

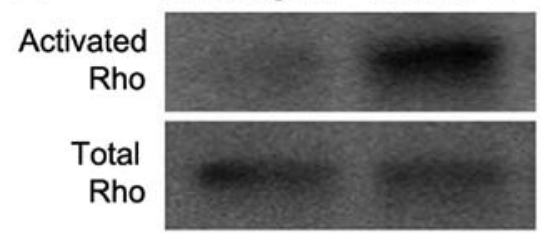

B

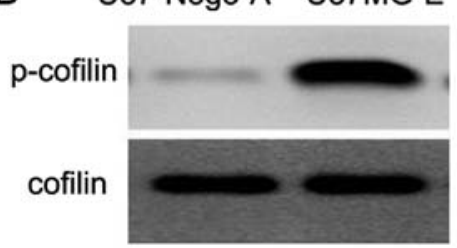

Figure 8. Expression of Rho and cofilin by western blotting. Representative results of the Rho activity assay (A) and cofilin (B).

Cytoskeletal actin/vimentin changes. Immunofluorescence staining of actin and vimentin was performed to evaluate whether the differences in cytoskeletal alterations were associated with the cell motility and invasion of malignant glioma cells. U87-Nogo-A cells became round and flat, while U87MG-E cells showed a bipolar shape. U87-Nogo-A cells with less motility showed fewer stress fibers, shorter lamellipodia and the cytoskeletal proteins were mainly concentrated around the nucleus and became entangled, compared with the U87MG-E cells (Fig. 7).

Nogo-A decreases Rho GTPase activity and cofilin phosphorylation. Rho proteins are well established factors which regulate cell shape and motility. We investigated Rho activity and expression of total Rho GTPase (RhoA, B and C) in the U87MG-E and U87-Nogo-A cells. Rho GTPases cycle between an active GTP-bound and an inactive GDP-bound conformation. In the GTP-bound state, Rho GTPases interact with downstream targets to elicit cellular responses. The U87-Nogo-A cells showed decreased GTP-bound Rho compared with the U87MG-E cells (Fig. 8A). Cofilin, an actin severing protein inactivated through phosphorylation, is a downstream factor of the Rho GTPases. In our results, phosphorylated cofilin was also decreased in the U87-Nogo-A cells, compared with the control (Fig. 8B).

\section{Discussion}

The complete removal of gliomas is usually microscopically impossible due to the insidious infiltration of tumor cells into the surrounding normal brain tissue. Glioma cells use myelinated nerve fiber tracts, vessel basement membranes and the subependymal layers as major routes for brain invasion (17). This is the primary cause of therapeutic failure.

Proteins of the reticulon family are membrane-bound proteins present in all eukaryotic organisms examined, and range in size from 200 to 1,200 amino acids. Vertebrate reticulons are attracting more attention due to their roles in various disorders including neurodegenerative diseases. The nomenclature of reticulons originally referred to a neuroendocrine-specific protein, anchored mainly on the membrane of smooth endoplasmic reticulum (18). 
In the adult CNS, Nogo-A is expressed in oligodendrocytes, in the inner and outer loops of myelin sheaths, and in some neuronal populations such as dorsal root ganglion cells, retinal ganglion cells and ventral horn neurons in the spinal cord $(5,7,19)$. During development, Nogo-A is expressed in neurons, where it has been suggested to play a role in neuronal migration and cortical development (20). In the present study, we identified that expression of Nogo-A was found in a higher percentage of oligodendrogliomas than in glioblastomas, using western blotting and IHC, in accordance with previous results (11). These results may indicate a correlation between Nogo-A expression and tumor grade. Nogo-A was found to have a negative relationship with the malignancy of oligodendroglial tumors (12) and furthermore, Nogo was found to enhance the adhesion of olfactory-ensheathing cells and to inhibit their migration (21). These results suggest that Nogo-A may be involved in the migration and invasion of glioblastoma cells.

In the present study, we investigated whether Nogo-A is associated with glioma cell migration and invasion. Firstly, we selected the U87MG cell line with high motility and no expression of Nogo-A. The 'U87-Nogo-A' cell line, continuously overexpressing Nogo-A and the 'U87MG-E' cell line as a control were established. The effect of Nogo-A on the migratory and invasive abilities was evaluated by an in vitro assay that involved the simple scratch technique and the Boyden chamber system. The results showed that the motility and invasion ability of the U87-Nogo-A cells were significantly decreased compared with the control cells.

Gliomas, even low grade gliomas, invade surrounding normal tissues at a very early stage. Cell migration involves multiple processes that are regulated by various signaling molecules (22), and incudes changes in the cytoskeleton, cell-substrate adhesion, and the extracellular matrix. The actin cytoskeleton and its regulatory proteins are crucial for cell migration in most cells. During cell migration, the actin cytoskeleton is dynamically remodeled, and this reorganization produces the force necessary for cell migration (23).

Thus, alterations in cytoskeletal proteins, actin and vimentin were examined by immunofluorescence staining in the U87-Nogo-A cells overexpressing Nogo-A. Expression patterns of actin and vimentin in the U87-Nogo-A cells were observed to concentrate around the nucleus, and the cells had fewer stress fibers and shorter lamellipodia.

Rho proteins play a role in organelle development, cytoskeletal dynamics, cell movement, and other common cellular functions. Rho family small GTPases play pivotal roles in the reorganization of the actin cytoskeleton during cell migration $(23,24)$. Higher vertebrates have 3 Rho GTPases (RhoA, RhoB and RhoC), which share $85 \%$ amino acid sequence identity. Although RhoB and RhoC were characterized at the same time as RhoA, RhoB and RhoC have received less attention due to their extensive homology with RhoA (25). Nogo-A inhibits neurite outgrowth through the $\mathrm{NgR}$ receptor, subsequently activating RhoA. RhoA is involved in diverse cellular functions including cytokinesis (26), cellular migration (27) and adhesion (28), and biological processes central to tumorigenesis. Increased RhoA expression has been demonstrated in a variety of malignancies (29-33) and the expression is pronounced in high-grade astrocytomas (34).
Cofilin, an actin depolymerization factor, is inactivated through phosphorylation downstream of RhoA signaling. It can directly affect the rate of actin polymerization, actin filopodia formation, growth cone motility $(35,36)$, and as recently shown, neurite outgrowth (37). Since Rho regulates the bundling of actin filaments into stress fibers and the formation of focal adhesion complexes (38), a decrease in Rho activity should influence cell stress fiber formation. A decrease in phosphorylated cofilin clearly leads to an increase in severing actin, followed by actin reorganization, and consequently cytoskeleton changes and alterations in cell migration and invasion.

Based on these studies, we aimed to ascertain whether Nogo-A-overexpressing cells have altered Rho activity and phosphorylated cofilin using western blotting. As expected, Rho activity and p-cofilin expression were decreased in the U87-Nogo-A cells, compared with the U87MG-E cells. These results suggest that migration and invasion decreased by overexpression of Nogo-A may be the result of changes in Rho activity in the U87MG malignant glioma cells.

In conclusion, Nogo-A negatively modulated the motility and invasion through regulation of RhoA and cofilin in the U87MG malignant glioma cell line. Since there are numerous downstream molecules which can influence cytoskeleton re-organization through the RhoA signaling pathway, further experiments must be performed to build upon our hypothesis.

\section{Acknowledgements}

The present study was supported by the Leading Foreign Research Institute Recruitment Program through the National Research Foundation of Korea (NRF) funded by the Ministry of Education, Science and Technology (MEST) (2011-0030034).

\section{References}

1. Maher EA, Furnari FB, Bachoo RM, Rowitch DH, Louis DN, Cavenee WK and DePinho RA: Malignant glioma: Genetics and biology of a grave matter. Genes Dev 15: 1311-1333, 2001.

2. Guo L, Qiu Y, Ge J and Zhou D: Glioblastoma multiforme with subcutaneous metastases, case report and literature review. J Korean Neurosurg Soc 52: 484-487, 2012.

3. Oertle T, Klinger M, Stuermer CA and Schwab ME: A reticular rhapsody: Phylogenic evolution and nomenclature of the RTN/Nogo gene family. FASEB J 17: 1238-1247, 2003.

4. GrandPré T, Li S and Strittmatter SM: Nogo-66 receptor antagonist peptide promotes axonal regeneration. Nature 417: 547-551, 2002.

5. Huber AB, Weinmann O, Brösamle C, Oertle T and Schwab ME: Patterns of Nogo mRNA and protein expression in the developing and adult rat and after CNS lesions. J Neurosci 22: 3553-3567, 2002.

6. Hunt D, Coffin RS, Prinjha RK, Campbell G and Anderson PN: Nogo-A expression in the intact and injured nervous system. Mol Cell Neurosci 24: 1083-1102, 2003.

7. Wang X, Chun SJ, Treloar H, Vartanian T, Greer CA and Strittmatter SM: Localization of Nogo-A and Nogo-66 receptor proteins at sites of axon-myelin and synaptic contact. J Neurosci 22: 5505-5515, 2002.

8. Josephson A, Widenfalk J, Widmer HW, Olson L and Spenger C: NOGO mRNA expression in adult and fetal human and rat nervous tissue and in weight drop injury. Exp Neurol 169: 319-328, 2001.

9. Niederöst B, Oertle T, Fritsche J, McKinney RA and Bandtlow CE: Nogo-A and myelin-associated glycoprotein mediate neurite growth inhibition by antagonistic regulation of RhoA and Rac1. J Neurosci 22: 10368-10376, 2002. 
10. Boureux A, Vignal E, Faure S and Fort P: Evolution of the Rho family of ras-like GTPases in eukaryotes. Mol Biol Evol 24: 203-216, 2007.

11. Kuhlmann T, Gutenberg A, Schulten HJ, Paulus W, Rohde V and Bruck W: Nogo-a expression in glial CNS tumors: A tool to differentiate between oligodendrogliomas and other gliomas? Am J Surg Pathol 32: 1444-1453, 2008.

12. Xiong NX, Zhao HY, Zhang FC and He ZQ: Negative correlation of Nogo-A with the malignancy of oligodendroglial tumor. Neurosci Bull 23: 41-45, 2007.

13. Jung TY, Jung S, Lee KH, Cao VT, Jin SG, Moon KS, Kim IY, Kang SS, Kim HS and Lee MC: Nogo-A expression in oligodendroglial tumors. Neuropathology 31: 11-19, 2011.

14. Schwab ME: Myelin-associated inhibitors of neurite growth and regeneration in the CNS. Trends Neurosci 13: 452-456, 1990

15. Teng FY and Tang BL: No go for brain tumors? J Mol Neurosci 25: $1-6,2005$.

16. Walker JM: The bicinchoninic acid (BCA) assay for protein quantitation. Methods Mol Biol 32: 5-8, 1994.

17. Giese A and Westphal M: Glioma invasion in the central nervous system. Neurosurgery 39: 235-252, 1996.

18. van de Velde HJ, Roebroek AJ, Senden NH, Ramaekers FC and Van de Ven WJ: NSP-encoded reticulons, neuroendocrine proteins of a novel gene family associated with membranes of the endoplasmic reticulum. J Cell Sci 107: 2403-2416, 1994.

19. Buss A, Sellhaus B, Wolmsley A, Noth J, Schwab ME and Brook GA: Expression pattern of NOGO-A protein in the human nervous system. Acta Neuropathol 110: 113-119, 2005.

20. Mingorance-Le Meur A, Zheng B, Soriano E and del Río JA: Involvement of the myelin-associated inhibitor Nogo-A in early cortical development and neuronal maturation. Cereb Cortex 17: 2375-2386, 2007.

21. Su Z, Cao L, Zhu Y, Liu X, Huang Z, Huang A and He C: Nogo enhances the adhesion of olfactory ensheathing cells and inhibits their migration. J Cell Sci 120: 1877-1887, 2007.

22. Ridley AJ, Schwartz MA, Burridge K, Firtel RA, Ginsberg MH, Borisy G, Parsons JT and Horwitz AR: Cell migration: Integrating signals from front to back. Science 302: 1704-1709, 2003.

23. Pollard TD and Borisy GG: Cellular motility driven by assembly and disassembly of actin filaments. Cell 112: 453-465, 2003.

24. Raftopoulou M and Hall A: Cell migration: Rho GTPases lead the way. Dev Biol 265: 23-32, 2004.
25. Yamazaki D, Kurisu S and Takenawa T: Regulation of cancer cell motility through actin reorganization. Cancer Sci 96: 379-386, 2005.

26. Glotzer M: The molecular requirements for cytokinesis. Science 307: 1735-1739, 2005.

27. Ridley AJ: Rho GTPases and cell migration. J Cell Sci 114: 2713-2722, 2001

28. Chrzanowska-Wodnicka $\mathrm{M}$ and Burridge $\mathrm{K}$ : Rho-stimulated contractility drives the formation of stress fibers and focal adhesions. J Cell Biol 133: 1403-1415, 1996.

29. Fritz G, Just I and Kaina B: Rho GTPases are over-expressed in human tumors. Int J Cancer 81: 682-687, 1999.

30. Horiuchi A, Imai T, Wang C, Ohira S, Feng Y, Nikaido T and Konishi I: Up-regulation of small GTPases, RhoA and RhoC, is associated with tumor progression in ovarian carcinoma. Lab Invest 83: 861-870, 2003.

31. Kamai T, Arai K, Tsujii $\mathrm{T}$, Honda $\mathrm{M}$ and Yoshida $\mathrm{K}$ : Overexpression of RhoA mRNA is associated with advanced stage in testicular germ cell tumour. BJU Int 87: 227-231, 2001.

32. Kamai T, Tsujii T, Arai K, Takagi K, Asami H, Ito Y and Oshima H: Significant association of Rho/ROCK pathway with invasion and metastasis of bladder cancer. Clin Cancer Res 9: 2632-2641, 2003

33. Liu N, Bi F, Pan Y, Sun L, Xue Y, Shi Y, Yao X, Zheng Y and Fan D: Reversal of the malignant phenotype of gastric cancer cells by inhibition of RhoA expression and activity. Clin Cancer Res 10: 6239-6247, 2004.

34. Yan B, Chour HH, Peh BK, Lim C and Salto-Tellez M: RhoA protein expression correlates positively with degree of malignancy in astrocytomas. Neurosci Lett 407: 124-126, 2006.

35. Bernard O: Lim kinases, regulators of actin dynamics. Int $\mathbf{J}$ Biochem Cell Biol 39: 1071-1076, 2007.

36. Endo M, Ohashi K, Sasaki Y, Goshima Y, Niwa R, Uemura T and Mizuno K: Control of growth cone motility and morphology by LIM kinase and Slingshot via phosphorylation and dephosphorylation of cofilin. J Neurosci 23: 2527-2537, 2003.

37. Endo M, Ohashi K and Mizuno K: LIM kinase and slingshot are critical for neurite extension. J Biol Chem 282: 13692-13702, 2007.

38. Ridley AJ: Rho GTPases and actin dynamics in membrane protrusions and vesicle trafficking. Trends Cell Biol 16: 522-529, 2006. 